\title{
HIV testing in colposcopy and termination of pregnancy services: a missed opportunity?
}

\author{
Aparna Briggs, ${ }^{1}$ David G Partridge, ${ }^{2}$ Sylvia M Bates ${ }^{3}$
}

\author{
'Specialist Registrar, Department \\ of Genitourinary Medicine, \\ The Royal Hallamshire Hospital, \\ Sheffield, UK \\ ${ }^{2}$ Specialist Registrar, \\ Department of Infectious \\ Diseases, The Royal Hallamshire \\ Hospital, Sheffield, UK \\ ${ }^{3}$ Consultant, Department of \\ Genitourinary Medicine, \\ The Royal Hallamshire Hospital, \\ Sheffield, UK
}

\section{Correspondence to \\ Dr Aparna Briggs, Department of Genitourinary Medicine, \\ The Royal Hallamshire Hospital, Glossop Road, \\ Sheffield S10 2JF, UK; \\ aparnabriggs@yahoo.co.uk}

Received 23 February 2011

Accepted 17 June 2011

Published Online First

11 August 2011

\begin{abstract}
Introduction The 2008 National HIV testing guidelines produced jointly by the British Association of Sexual Health and HIV, British HIV Association and British Infection Society recommend HIV testing for patients attending termination of pregnancy (TOP) services and patients diagnosed with cervical intraepithelial neoplasia (CIN) Grade 2 or above. The aim is to reduce the time between acquisition and diagnosis of HIV by encouraging testing in settings where patients present with indicator diseases. Benefits of earlier HIV diagnosis include improved survival, prevention of onward transmission, and optimisation of maternal health when planning pregnancy. There is evidence that HIV reduces the effectiveness of standard treatment for CIN 2/3 and cervical cancer. The experience of antenatal screening indicates that the majority of women accept HIV screening if it is offered as part of a package of care.
\end{abstract}

Methods This retrospective case notes review of 60 HIV-positive women, diagnosed between 1 January 2006 and 31 July 2009, collected data on age, ethnicity, length of time in the UK, timing of HIV diagnosis and possible timing of acquisition relative to attendance at colposcopy or TOP services, CD4 count and symptoms at diagnosis and cervical cytology history. Results The authors found that three (5\%) women were diagnosed with CIN Grade 2 or above prior to HIV diagnosis; HIV testing at the time of TOP may have resulted in earlier diagnosis for three (5\%) women. There was at least one missed opportunity for earlier diagnosis in five (8\%) cases.

Conclusions The authors suggest further work should be undertaken to establish HIV prevalence in TOP and colposcopy services and that HIV testing should become standard practice in the management of CIN 2/3 and cervical cancer.

\section{Introduction}

ThecurrentNationalHIVTestingGuidelines produced jointly by the British Association

\section{Key message points \\ Knowledge of HIV status is relevant in the management of cervical intraepithelial neoplasia Grade 2/3. \\ - HIV testing is acceptable to women if offered as part of a package of care. \\ - Routine HIV testing is cost effective if prevalence exceeds 2 in 1000 and earlier testing is associated with improved clinical outcome.}

of Sexual Health and HIV, British HIV Association and British Infection Society published in 2008 recommend routine HIV testing for all patients attending termination of pregnancy (TOP) services and patients diagnosed with a range of specific indicator conditions including cervical cancer, cervical intraepithelial neoplasia (CIN) Grade 2 or above and vulval intraepithelial neoplasia. The principles of these guidelines have been endorsed by the subsequently published National Institute for Health and Clinical Excellence guidelines on increasing HIV testing. ${ }^{1}$ The aim is to decrease undiagnosed HIV infection and thereby reduce the proportion of HIV-positive patients diagnosed late in the course of the illness by expanding HIV testing to clinicians in specialities where patients may present with indicator diseases. An estimated one quarter of individuals living with HIV in the UK remain undiagnosed. ${ }^{2}$ The benefits of earlier diagnosis in HIV include decreased morbidity and mortality, improved treatment response, decreased health care costs and reduced onward transmission. On a population level, routine HIV testing is thought to be cost effective if prevalence exceeds 2 in 1000.

Currently, routine HIV testing is not offered in TOP and colposcopy services and there are few data on the prevalence of HIV in these settings. The Royal College of Obstetricians and Gynaecologists do not recommend routine HIV testing in TOP services and the Advisory Committee for Cervical Screening (British Society for 
Colposcopy and Cervical Pathology to Gynaecologists/ Colleagues communication, 15 March 2011) dispute the need or appropriateness of HIV testing in colposcopy. This study aimed to determine the proportion of women diagnosed with HIV between 1 January 2006 and 31 July 2009 at our service who potentially could have been diagnosed earlier if HIV testing had been routinely offered in these services.

\section{Methods}

Data from 60 women diagnosed with HIV between 1 January 2006 and 31 July 2009 were included in a retrospective case note review. This was a convenience sample of the first 30 of 43 accessible notes in chronological order of diagnosis from the Department of Genitourinary Medicine and the first 30 of 88 alphabetically ordered from the Department of Infectious Diseases. Data were collected on age, ethnicity, length of time in the UK, timing of HIV diagnosis relative to attendance at colposcopy or TOP services, CD4 count and symptoms at diagnosis, possible timing of HIV acquisition based on previous negative tests, contact tracing or possible seroconversion illness, and cytology history. All cases were cross-referenced using the laboratory APEX ${ }^{\mathrm{TM}}$ database that contains all cytology and histology results for samples taken within the Sheffield area.

\section{Results}

Of 60 women, 43 (72\%) were born in an African country and $10(17 \%)$ were born in the UK. Ten (17\%) were below the age at which routine cervical screening is offered. Median age at diagnosis was 33 (range 21-60) years. Cytology history was available for 46 women and of these 31 (67\%), including two under 25 years of age, had had at least one cervical cytology test prior to HIV diagnosis. Three $(5 \%)$ had a diagnosis of CIN Grade 2 or above prior to the HIV diagnosis, of whom two were Black African and one was White British. They ranged in age from 28 to 60 years. By the time of HIV diagnosis, two of these women had CD4 counts below the treatment threshold of 350 , one of whom presented ill with an AIDS-defining illness.

Of 60 women, six (10\%) had a history of TOP prior to diagnosis. In 3/60 (5\%) cases HIV testing at the time of TOP may have resulted in earlier diagnosis; in one (2\%) case timing of TOP was unknown; in the other two cases TOP likely preceded the acquisition of HIV. Of the three who may have benefited from HIV testing two were Black African and one was White British. At the time of HIV diagnosis two had CD4 counts below 350, one of whom presented ill with an AIDS-defining illness.

One woman had two prior missed opportunities for diagnosis, once in TOP services and once in colposcopy. Therefore 5/60 (8\%) patients, had at least one missed opportunity for earlier diagnosis in women's services, with delay in diagnosis ranging between 3 and 40 months. Two of these five women had no apparent traditional risk factors for HIV.

\section{Discussion}

Despite $17 \%$ of our sample being below the age at which routine cervical screening is offered and only $67 \%$ having definitely had a previous cervical cytology, we found that three $(5 \%)$ had an opportunity for earlier diagnosis if following the 2008 national guidelines regarding testing in colposcopy. This figure and the overall figure of five $(8 \%)$ women when previous TOP is included are likely to be underestimates as events not documented in the history would be missed and cytology/colposcopy performed outside the Sheffield area would not be recorded on APEX. Women may not disclose a previous TOP unless specifically asked.

The natural history of human papillomavirus (HPV)related cervical disease in women with HIV has been well documented. There are differences in the prevalence, incidence, progression and regression of cervical lesions. The risk and severity of the lesions correlate with the degree of immunodeficiency. A recent analysis of the French Hospital Database of HIV cohort concluded that immunodeficiency had a major role in HPV-related cervical cancers and the risk was reduced by antiretroviral therapy (ART) even when controlled for CD4 count. ${ }^{3}$

In addition, knowledge of a patient's HIV status may influence management of $\mathrm{CIN}$ as evidence suggests that standard excisional treatments for CIN are associated with higher rates of recurrence in HIV-positive women. The recurrence rate of CIN after excision with negative margins has been estimated at 20-75\% compared with $8.5-16 \%$ in those not known to be HIV-positive. ${ }^{4}$ The risk of recurrence may be reduced by exposure to appropriate ART.

HIV-positive women are also more likely to have involved margins after conisation. ${ }^{5}$ Knowledge of a woman's HIV status may, therefore, affect the management and prognosis of cervical lesions.

There are few published studies examining HIV prevalence or testing in populations of women with HPV-related cervical disease. Prior to the availability of effective treatment in the form of highly active antiretroviral therapy, acceptance rates of HIV testing were found to be $50-60 \%{ }^{67}$ Data from New York showed HIV seroprevalence in colposcopy clinics was higher than in both antenatal clinics and the general population: $4 \%$ overall prevalence of undiagnosed HIV (6.1\% in those accepting a test) compared with an estimated $1.24 \%$ of women of childbearing age and $1.6 \%$ of obstetric patients. ${ }^{8}$ No more recent data have been identified on literature review.

Data on HIV testing in TOP services are scarce. Published studies from the USA ${ }^{9}$ and data from London report rates of between $0.1 \%$ and $0.87 \%$ with a testing acceptance rate of $>96 \% .{ }^{10}$ Prevalence of HIV among pregnant women in 2008 was $0.15 \%$ in England (excluding London) and $0.39 \%$ in London [Health Protection Agency (HPA) data] but very few data are available on the prevalence of HIV in patients attending colposcopy clinics and TOP services across the UK. 
Uptake of HIV testing in antenatal clinics exceeded 95\% in 2009 (HPA data ${ }^{2}$ ) and is more than $98.5 \%$ in Sheffield (internal audit), suggesting that a high uptake of HIV testing is achievable. The Department of Health funded eight projects looking at HIV testing in a variety of hospital departments, primary care and community settings. ${ }^{10}$ This report concluded that expanded HIV testing in primary care, general medical admissions and in community settings targeting high-risk populations is acceptable and feasible. A point-of-care test was shown to be helpful in primary care settings as it reduced the administrative burden of handling negative results.

Early identification of HIV infection is important as timely initiation of ART leads to dramatically better survival and reduced morbidity compared with delayed initiation. In addition it is likely to reduce the onward transmission of HIV. Acceptability of HIV testing should be higher now that appropriate treatment is available and it is cost effective even when prevalence of HIV is low. Clinician-related barriers to uptake of HIV testing may be significant as suggested by the HINTS study. ${ }^{11}$ There is evidence that HIV reduces the effectiveness of standard treatment for CIN 2/3 and cervical cancer. We suggest that further work should be undertaken to look at HIV prevalence in TOP and colposcopy services and that HIV testing should become standard practice in the treatment of CIN 2/3 and cervical cancer.

Acknowledgements The authors thank Professor G Kinghorn for his advice.

Competing interests None.

Provenance and peer review Not commissioned; externally peer reviewed.

\section{References}

1 National Institute for Health and Clinical Excellence. PH33 Increasing the Uptake of HIV Testing Among Black Africans in
England: Guidance. 2011. http://guidance.nice.org.uk/PH33/ Guidance/pdf/English [accessed 5 May 2011].

2 Health Protection Agency. HIV in the United Kingdom: 2010 Report. 2010. http://www.hpa.org.uk/web/ HPAweb\&HPAwebStandard/HPAweb_C/1287145264558 [accessed 5 May 2011].

3 Guiguet M, Boué F, Cadranel J, et al.; Clinical Epidemiology Group of the FHDH-ANRS CO4 cohort. Effect of immunodeficiency, HIV viral load, and antiretroviral therapy on the risk of individual malignancies (FHDH-ANRS CO4): a prospective cohort study. Lancet Oncol 2009;10: 1152-1159.

4 Tebeu PM, Major AL, Mhawech P, et al. The recurrence of cervical intraepithelial neoplasia in HIV-positive women: a review of the literature. Int J STD AIDS 2006;17:507-511.

5 Gilles C, Manigart Y, Konopnicki D, et al. Management and outcome of cervical intraepithelial neoplasia lesions: a study of matched cases according to HIV status. Gynecol Oncol 2005;96:112-118.

6 Jennings TS, Dottino P, Sperling R, et al. Screening for human immunodeficiency virus in inner city females with abnormal cervical cytology. Infect Dis Obstet Gynecol 1996;4:281-286.

7 McCarthy KH, Johnson MA, Lawton FG, et al. Acceptability of screening for HIV seroprevalence in women with cervical pathology. Lancet 1992;340:1040-1041.

8 Spitzer M, Brennessel D, Seltzer VL, et al. Is human papillomavirus-related disease an independent risk factor for human immunodeficiency virus infection? Gynecol Oncol 1993;49:243-246.

9 Drey EA, Darney PD, Louie B, et al. HIV sentinel surveillance among women seeking elective pregnancy termination, San Francisco. Sex Transm Dis 2005;32:590-592.

10 Crowe G, Chibber F. Voluntary named HIV testing amongst women undergoing termination of pregnancy is feasible and acceptable. HIV Med 2008;9(Suppl. 1):25.

11 Health Protection Agency. Time to Test for HIV: Expanded Healthcare and Community Testing in England, Interim Report. 2010. http://www.hpa.org.uk/web/HPAweb\&HPAwebStandard/ HPAweb_C/1287145269283 [accessed 5 May 2011]. 